\title{
The Resistance Quantity in Knee Extension Movement of Exercise Bands (Thera-Band ${ }^{\circledR}$ )
}

\author{
Noboru SAKANOUe, RPT, $\mathrm{PhD}^{1)}$, KUnihiro KatAyama, RPT, MS ${ }^{2)}$ \\ 1)Department of Physical Therapy, Faculty of Health Science, Ryotokuji University: 5-8-1 \\ Akemi, Urayasu, Chiba 279-8567, Japan. TEL: +81 47-382-2111, \\ E-mail:no-sakanoue@ryotokuji-u.ac.jp \\ ${ }^{2)}$ Department of Physical Therapy, Kochi Rehabilitation Institute: 1139-3 Otsu Takaoka, Tosa, \\ Kochi 781-1102, Japan.
}

\begin{abstract}
This study was carried out for the purpose of quantifying resistance demonstrated by TheraBand $^{\circledR}$ exercise bands in knee joint extension. The subjects were 35 healthy adults ( 19 male, 16 female), and their average age was $21.0 \pm 2.3$ years old. The exercise bands had 6 colors. From the lower leg nutation position in sitting on the edge of a bed, knee joints were extended to $30^{\circ}$ and $45^{\circ}$, and the value of the muscle strength used in retaining those angles with the different colored exercise bands was measured with a dynamometer. In this study, the resistance demonstrated in knee extension with the exercise bands was quantified using a dynamometer, and the resistance quantity of the six colored exercise bands used in this study was clarified, enabling the construction of an index for their selection.
\end{abstract}

Key words: Exercise Bands, Resistance training, Resistance quantity

(This article was submitted May 21, 2007, and was accepted Aug. 3, 2007)

\section{INTRODUCTION}

Tubes and bands of elastic material are instruments which are often used for resistance training, for the purpose of muscle strengthening and the improvement of functional performance of sports players ${ }^{1)}$, for rehabilitation in orthopedic diseases $^{2)}$ and for the prevention of muscle strength decline in older adults ${ }^{3)}$. The resistance forces of tubes and bands are usually presented by the manufacturer, and made into an index for application to subjects. However, these values are not those measured by the application of the tube or band to the joint movement of the actual human body, and therapists subjectively and experientially select bands which are suitable for a subject.

This study was carried out for the purpose of quantifying the resistance demonstrated by TheraBand $^{\circledR}$ exercise bands (Ex-Bands) in knee joint extension.

\section{METHODS}

The subjects were 35 healthy adults (19 male, 16 female). The subjects had an average age of $21.0 \pm$ 2.3 years old, an average height of $164.7 \pm 8.7 \mathrm{~cm}$, an average weight of $59.0 \pm 10.1 \mathrm{~kg}$, and an average BMI of $21.6 \pm 2.6$ (Table 1). The purpose and method of this study were explained to the subjects and their consent to participation was obtained.

Six types of Ex-Bands (THIN [yellow], MEDIUM [red], HEAVY [green], EXTRA HEAVY [blue], SPECIAL HEAVY [black] and SUPER HEAVY [silver]) were used. Each ExBand was new out of the box, and was cut to $1 \mathrm{~m}$ in length. In consideration of the "prestretching effect" "), prior to the measurement with the first subject, the tester extended each Ex-Band about 20 times.

A hand-held dynamometer (Tas MF-01, Anima Corp.) was used for the measurement of the 
resistance of the Ex-Bands. The measurement position was sitting on the edge of a bed for treatment without a backrest, with the lower leg hanging off the bed in the leg-off bed position. The sensor of the dynamometer was placed just above the anterior surface of the ankle joint. The ExBands were cut to $1 \mathrm{~m}$, and made into a loop of 60 $\mathrm{cm}$ with a fastener. One end of the loop was held by the bed post, and the other was placed over the sensor of the dynamometer. The measurement was made at knee extension angles of $30^{\circ}$ and $45^{\circ}$ from the lower leg nutation position. The measurement assistant measured the joint angle by a goniometer, and each subject was told to retain the angle (Fig. 1). The dynamometer measurement was started from the time when joint angle maintenance was possible, and the value was recorded. The measurement at each angle was carried out 2 times, and the mean value was adopted as the measured value. The measurement was repeated, if Ex-Bands came off the sensor unit when the knee joint was extended.

Basic statistics were calculated. A one-way analysis of variance (ANOVA) and post-hoc test were performed to examine the measured value divided by the subject's height. A two-tailed $t$ test was performed to examine the measured values between males and females. Statistical significance for all tests performed in this study was defined as $\mathrm{p}<0.05$.

\section{RESULTS}

The resistance quantity of knee extensions at $30^{\circ}$ and $45^{\circ}$ of each Ex-Band is shown in Table 2.

We classified the subjects into 3 groups of 150 $\mathrm{cm}$ (mean height; $154.9 \pm 3.0 \mathrm{~cm}$ ), $160 \mathrm{~cm}$ (mean height; $164.8 \pm 2.5 \mathrm{~cm}$ ) and $170 \mathrm{~cm}$ (mean height; $175.5 \pm 4.4 \mathrm{~cm}$ ) by height, and examined the measured values of the 3 groups. There was a statistically significant difference between the 150 $\mathrm{cm}$ and $170 \mathrm{~cm}$ groups in use of the yellow $(\mathrm{p}<0.01)$ and red $(\mathrm{p}<0.05)$ Ex-Bands at knee extension of $30^{\circ}$, and between the $160 \mathrm{~cm}$ and $170 \mathrm{~cm}$ groups in use of the yellow $(p<0.05)$ and red $(p<0.05)$ ExBands at knee extensions of $30^{\circ}$ and $45^{\circ}$ (Table 3 ). However, no other statistically significant differences were found at the measured values between each other groups.

The resistance quantity of knee extensions of $30^{\circ}$ and $45^{\circ}$ for each Ex-Band is shown for males and
Table 1. Characteristics of subjects

\begin{tabular}{lcc}
\hline & \multicolumn{2}{c}{ All subjects $(\mathrm{n}=35)$} \\
& Male $(\mathrm{n}=19)$ & Female $(\mathrm{n}=16)$ \\
\hline Age (years) & $21.4 \pm 3.0$ & $21.0 \pm 2.3$ \\
& \multicolumn{2}{c}{$164.7 \pm 8.7$} \\
Height $(\mathrm{cm})$ & $170.7 \pm 6.3$ & $157.6 \pm 4.8$ \\
Body weight $(\mathrm{kg})$ & $65.2 \pm 7.5$ & $51.6 \pm 7.5$ \\
& \multicolumn{2}{c}{$59.0 \pm 10.1$} \\
BMI & $22.4 \pm 2.6$ & $20.7 \pm 2.2$ \\
\hline
\end{tabular}

Values are mean \pm SD.

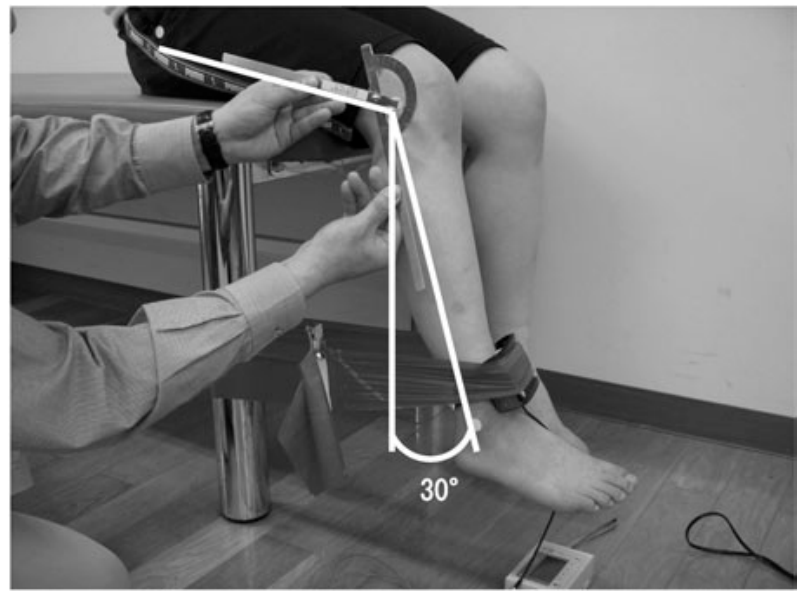

Fig. 1. Measurement of the resistance of Ex-Bands. The figure shows the measurement of resistance at knee extension $30^{\circ}$ from the lower leg nutation position.

females in Table 4. In a comparison of the measured values of males and females for each ExBand at each knee extension angle, no significant differences could be found.

\section{DISCUSSION}

In this study, the resistance of Ex-Bands demonstrated in knee joint extension was quantified using a dynamometer. It is considered that the resistance of the six colored Ex-Bands (TheraBand $^{\circledR}$ ) measured in this study was clarified, enabling the construction of an index for the selection of Ex-Bands.

Patterson et al. ${ }^{4)}$ used the same 6 colors of TheraBand tubing as this study to plot the resistance chart 
Table 2. Resistance of each Ex-Band in knee extensions of $30^{\circ}$ and $45^{\circ}$

unit: $\mathrm{kgf}$

\begin{tabular}{lcc}
\hline & Knee extension $30^{\circ}$ & Knee extension $45^{\circ}$ \\
\hline THIN (yellow) & $4.2 \pm 0.3$ & $4.7 \pm 0.3$ \\
MEDIUM (red) & $4.6 \pm 0.3$ & $5.1 \pm 0.4$ \\
HEAVY (green) & $5.7 \pm 0.4$ & $6.3 \pm 0.4$ \\
EXTRA HEAVY (blue) & $6.7 \pm 0.4$ & $7.4 \pm 0.5$ \\
SPECIAL HEAVY (black) & $7.6 \pm 0.5$ & $8.5 \pm 0.5$ \\
SUPER HEAVY (silver) & $11.3 \pm 0.6$ & $12.8 \pm 0.7$ \\
\hline
\end{tabular}

Values are mean \pm SD.

Table 3. Resistance of each Ex-Band by subject height grouping in knee extensions of $30^{\circ}$ and $45^{\circ}$

\begin{tabular}{|c|c|c|c|c|c|c|}
\hline & \multicolumn{3}{|c|}{ Knee extension $30^{\circ}$} & \multicolumn{3}{|c|}{ Knee extension $45^{\circ}$} \\
\hline & $\begin{array}{l}150 \mathrm{~cm} \\
(\mathrm{n}=11)\end{array}$ & $\begin{array}{l}160 \mathrm{~cm} \\
(\mathrm{n}=14)\end{array}$ & $\begin{array}{l}170 \mathrm{~cm} \\
(\mathrm{n}=10)\end{array}$ & $\begin{array}{l}150 \mathrm{~cm} \\
(\mathrm{n}=11)\end{array}$ & $\begin{array}{l}160 \mathrm{~cm} \\
(\mathrm{n}=14)\end{array}$ & $\begin{array}{l}170 \mathrm{~cm} \\
(\mathrm{n}=10)\end{array}$ \\
\hline THIN (yellow) & $\begin{array}{c}4.0 \pm 0.2 \\
\llcorner\end{array}$ & $\begin{array}{c}4.2 \pm 0.3 \\
* *\end{array}$ & $4.4 \pm 0.3$ & $4.6 \pm 0.2$ & $4.6 \pm 0.3$ & $4.9 \pm 0.4$ \\
\hline MEDIUM (red) & $4.5 \pm 0.2$ & $\begin{array}{c}4.5 \pm 0.4 \\
\llcorner\end{array}$ & $4.8 \pm 0.3$ & $5.1 \pm 0.2$ & $5.0 \pm 0.4$ & $5.3 \pm 0.4$ \\
\hline HEAVY (green) & $5.5 \pm 0.2$ & $5.7 \pm 0.4$ & $5.8 \pm 0.3$ & $6.3 \pm 0.3$ & $6.2 \pm 0.4$ & $6.6 \pm 0.4$ \\
\hline EXTRA HEAVY (blue) & $6.6 \pm 0.2$ & $6.6 \pm 0.5$ & $6.8 \pm 0.2$ & $7.3 \pm 0.3$ & $7.3 \pm 0.5$ & $7.7 \pm 0.4$ \\
\hline SPECIAL HEAVY (black) & $7.4 \pm 0.3$ & $7.6 \pm 0.5$ & $7.8 \pm 0.4$ & $8.4 \pm 0.3$ & $8.4 \pm 0.6$ & $8.8 \pm 0.5$ \\
\hline SUPER HEAVY (silver) & $10.9 \pm 0.3$ & $11.5 \pm 0.6$ & $11.4 \pm 0.8$ & $12.4 \pm 0.5$ & $13.0 \pm 0.7$ & $12.8 \pm 0.7$ \\
\hline
\end{tabular}

Values are mean \pm SD.

ANOVA and post-hoc (Tukey) test: $*=\mathrm{p}<0.05, * *=\mathrm{p}<0.01$

for extension up to $250 \%$. They reported the material displays a non-linear behavior in the initial stretch phase and a linear behavior after $50 \%$ elongation as the characteristics. Tafel et al. ${ }^{5}$ ) reported the loads recorded when exercise tubes of 5 colors, $12 \mathrm{~cm}$ in length, were extended to $100 \%$, $200 \%$ and $300 \%$. Representative values at extension of $100 \%$ were: yellow $0.6 \pm 0.1 \mathrm{kgf}$, red $2.3 \pm 0.1 \mathrm{kgf}$, green $2.7 \pm 0.1 \mathrm{kgf}$, blue $3.1 \pm 0.1 \mathrm{kgf}$, black $3.6 \pm 0.1 \mathrm{kgf}$. Simoneau et al. ${ }^{6)}$ measured the tension in extending yellow, green and black elastic bands and elastic tubing at $100 \%$ and $200 \%$ extension of length. They reported tensions for $100 \%$ elongation of bands: yellow $14.4 \pm 0.4 \mathrm{~N}$, green $27.8 \pm 0.6 \mathrm{~N}$, and black $39.1 \pm 1.0 \mathrm{~N}$; for $100 \%$ elongation of tubing: yellow $4.2 \pm 0.2 \mathrm{~N}$, green $21.9 \pm 0.3 \mathrm{~N}$, and black $34.2 \pm 0.3 \mathrm{~N}$; for $200 \%$ elongation of bands: yellow $20.4 \pm 1.1 \mathrm{~N}$, green $37.1 \pm 3.2 \mathrm{~N}$, and black $58.1 \pm 5.5 \mathrm{~N}$; and for $200 \%$ elongation of tubing: yellow $6.7 \pm 0.2 \mathrm{~N}$, green $34.3 \pm 0.5 \mathrm{~N}$, and black $51.4 \pm 2.7 \mathrm{~N}$.

When tubes or bands are used for resistance training of the lower extremities, they must be attached to the moving limb after fixation to a treatment table. Therefore, they are often used as a loop. However, the above noted researches recorded the resistance force by stretching both ends of a tube or band cut off at set length in a machine. In contrast, this study quantified the resistance quantity by carrying out the research in a condition equivalent to one in which resistance training of knee extensor muscles actually takes place. Therefore, it is difficult to compare the results of the earlier studies with the results of this study. Additionally, there seems to be a problem in applying the results of the preceding research to an index for the resistance force in knee extension training. Also, the material used in this study was a flat band, Thera-Band. In the research of Simoneau et al. ${ }^{6}$, it is reported that the tension of tubing shows a lower value than the tension of a band, therefore, it is thought that the results of this study cannot be applied to Thera-Band tubing.

In the result of this study, for the variation of 
Table 4. Resistance of each Ex-Band by gender in knee extensions of $30^{\circ}$ and $45^{\circ}$

\begin{tabular}{|c|c|c|c|c|}
\hline & \multicolumn{2}{|c|}{ Knee extension $30^{\circ}$} & \multicolumn{2}{|c|}{ Knee extension $45^{\circ}$} \\
\hline & $\begin{array}{l}\text { Male } \\
(n=19)\end{array}$ & $\begin{array}{c}\text { Female } \\
(\mathrm{n}=16)\end{array}$ & $\begin{array}{l}\text { Male } \\
(n=19)\end{array}$ & $\begin{array}{c}\text { Memale } \\
(\mathrm{n}=16)\end{array}$ \\
\hline THIN (yellow) & $4.3 \pm 0.3$ & $4.2 \pm 0.3$ & $4.7 \pm 0.4$ & $4.7 \pm 0.3$ \\
\hline MEDIUM (red) & $4.6 \pm 0.4$ & $4.6 \pm 0.3$ & $5.1 \pm 0.4$ & $5.1 \pm 0.2$ \\
\hline HEAVY (green) & $5.6 \pm 0.4$ & $5.7 \pm 0.3$ & $6.3 \pm 0.5$ & $6.4 \pm 0.3$ \\
\hline EXTRA HEAVY (blue) & $6.6 \pm 0.4$ & $6.7 \pm 0.3$ & $7.4 \pm 0.5$ & $7.5 \pm 0.4$ \\
\hline SPECIAL HEAVY (black) & $7.5 \pm 0.4$ & $7.6 \pm 0.5$ & $8.5 \pm 0.6$ & $8.5 \pm 0.4$ \\
\hline SUPER HEAVY (silver) & $11.3 \pm 0.7$ & $11.2 \pm 0.6$ & $12.8 \pm 0.7$ & $12.7 \pm 0.7$ \\
\hline
\end{tabular}

Values are mean $\pm \mathrm{SD}$.

No statistically significant differences were found between males and females at knee extension angles of $30^{\circ}$ and $45^{\circ}$.

resistance among the four colors of red, green, blue and black in knee extension angles of $30^{\circ}$ and $45^{\circ}$, the resistance quantity increased progressively with change of color at an amount of about $1 \mathrm{kgf}$ (Table $2)$. In contrast, the increase of resistance between the yellow and red bands was not as large (about 0.4 kgf), while the increase of resistance between the silver and black bands was large (about $4 \mathrm{kgf}$ ). In the results of earlier studies ${ }^{4,5,7)}$, differences in the amount of change of resistance between colors was also shown, but they showed a slightly different tendency to the results of this study. Hughes et al. ${ }^{7}$ ) reported that the mechanical strains were a function of the thickness of material used for each color of tubing. There is difference between a tube and a band, but it is considered that the tendency shown in this study was also based on the thickness of the material. Therefore, we consider that therapists must recognize this difference between level/color characteristics in changing to an appropriate ExBand.

The moment arm is given as one of the factors which affects the resistance of Ex-Bands used to hold, or attached to, the exercise/moving limbs. ExBands would be located more distal even when the joint angle is equal, if the moment arm is long. Therefore, Ex-Bands would be extended longer, and the resistance would increase more due to the physical characteristics of rubber. The subjects were classified into 3 groups by height instead of the moment arm length in this study, and the measured values were examined. Significant differences were recognized between four conditions, and while significant differences were not found, in comparison with the average measured values of the $150 \mathrm{~cm}$ and $170 \mathrm{~cm}$ groups at knee extensions of $30^{\circ}$ and $45^{\circ}$, the values of the $170 \mathrm{~cm}$ group showed a tendency to be larger than the values of the $150 \mathrm{~cm}$ group. We speculate that this is an effect of the moment arm length. Lower leg length of the subjects was a mean $36.6 \pm 2.4 \mathrm{~cm}$, and there was significant and strong correlation $(r=0.901, p<0.01)$ between the lower leg length and the height. The lower leg lengths of each group were $34.2 \pm 1.5 \mathrm{~cm}$ for the $150 \mathrm{~cm}$ group, $36.6 \pm 1.3$ $\mathrm{cm}$ for the $160 \mathrm{~cm}$ group, and $39.1 \pm 1.2 \mathrm{~cm}$ for the $170 \mathrm{~cm}$ group, and the difference of lower leg length between the $150 \mathrm{~cm}$ and $170 \mathrm{~cm}$ groups was about $5 \mathrm{~cm}$. Therefore, we think that the effect of the moment arm should be considered when using Ex-Bands on subjects whose height differs by 20 $\mathrm{cm}$.

As for gender, there was not much difference in the measured values for each color of band at both angles of both groups, and statistical significance was not found. We mentioned above that it is necessary to consider the influence of moment arm length with height difference. However, the difference in mean height between males and females was $13.1 \mathrm{~cm}$, but the difference did not seem to influence the moment arm length. This fact seems to suggest that resistance of Ex-Bands is almost equal regardless of gender, if the physical characteristics of the subjects are similar.

Preceding studies on elastic tubing have reported resistance against/with elongation ratio, but this study considered the application in a clinical setting and determined resistance with joint angle variation. The values obtained by this study represent an index of resistance for resistance 
training of knee extensor muscles using Ex-Bands, and we regard it as applicable to the appropriate selection of Ex-Bands for individual use. Also, under the same conditions, as used in this study, ExBands could be utilized as a simple assessment index of muscle strength.

A limitation of this study is that we made no examination from the electromyographic and dynamic viewpoint, an issue which we would like to pursue in future research.

\section{REFERENCES}

1) Treiber FA, Lott J, Duncan J, et al.: Effects of Theraband and lightweight dumbbell training on shoulder rotation torque and serve performance in college tennis players. Am J Sports Med, 1998, 26: 510-515.

2) Hintermeister RA, Bey MJ, Lange GW, et al.:
Quantification of elastic resistance knee rehabilitation exercises. JOSPT, 1998, 28: 40-50.

3) Mikesky AE, Topp R, Wigglesworth JK, et al.: Efficacy of a home-based training program for older adults using elastic tubing. Eur J Appl Physiol, 1994, 69: 316-320.

4) Patterson RM, Stegink Jansen CW, Hogan HA, et al.: Material properties of Thera-Band Tubing. Phys Ther, 2001, 81: 1437-1445.

5) Tafel JA, Thacker JG, Hagemann JM, et al.: Mechanical performance of exertubing for isotonic hand exercise. J Burn Care Rehabil, 1987, 8: 333-335.

6) Simoneau GG, Bereda SM, Sobush DC, et al.: Biomechanics of elastic resistance in therapeutic exercise programs. J Orthop Sports Phys Ther, 2001, 31: 16-24.

7) Hughes CJ, Hurd K, Jones A, et al.: Resistance properties of Thera-Band ${ }^{\circledR}$ tubing during shoulder abduction exercise. JOSPT, 1999, 29: 413-420. 